Boletín de la Sociedad Geológica Mexicana

VOLUMEN 63, NÚM. 2, 2011, P. 345-352

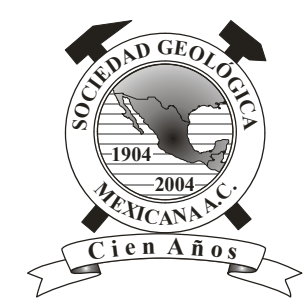

\title{
Determinación del esfuerzo de cedencia para suelos vulnerables a movimientos de remoción de masa originados por las lluvias
}

\author{
Arturo F. Méndez-Sánchez ${ }^{1, *}$, Leonor Pérez-Trejo ${ }^{1}$, Patricia Sánchez-Cruz ${ }^{1}$, \\ Ana Ma. Paniagua Mercado ${ }^{1}$ \\ ${ }^{1}$ Escuela Superior de Física y Matemáticas, Instituto Politécnico Nacional, Edif. 9 Unidad Profesional Adolfo López Mateos, Col. \\ Lindavista, C. P. 07738, México Distrito Federal, México. \\ *arturo@esfm.ipn.mx
}

\section{Resumen}

En el presente trabajo se estudia el esfuerzo de cedencia en función del contenido de agua para suelos propensos a flujos de detritos debido a las lluvias. El esfuerzo de cedencia es calculado de la relación y metodología propuesta por Pashias et al. (1996), a partir de una prueba de asentamiento en geometría cilíndrica. La ventaja de emplear este método es que la determinación del esfuerzo de cedencia se realiza de manera fácil y rápida. Incluso, se puede realizar in situ en la zona susceptible a movimientos de remoción de masa. Se presentan resultados de la variación del esfuerzo de cedencia en función del contenido de agua para la zona del cerro de Xico localizada en Valle de Chalco, Estado de México, la cual es considerada como vulnerable a deslaves por las autoridades locales.

Palabras clave: Esfuerzo de cedencia, movimientos de remoción de masa, prueba de asentamiento.

\begin{abstract}
Yield stress as a function of water content for soils prone to debris flows due to rain is studied in this work. Yield stress is calculated by the slump test in cylindrical geometry by using the relation proposed by Pashias et al. (1996). The advantage of using this method is the quick and easy determination of yield stress. In this case, the test can be done in situ in the zone susceptible to debris flows. Results of yield stress variation as a function of water content are shown for the cerro de Xico zone located in Chalco Valley in México State, which is considered to be vulnerable to landslides by the local authorities.
\end{abstract}

Keywords: Yield stress, debris flows, slump test. 


\section{Introducción}

Los movimientos de remoción de masa, derrumbes o caídas, son fenómenos muy destructivos debido a la cantidad de material que transportan, principalmente tierra y lodo. Entre los factores principales que dan lugar a los movimientos de remoción de masa destacan,la topografía de la región, la erosión de la zona (Bisson et al., 2005; VanDine et al., 2005; Collins, 2008), la saturación de agua que resulta en las masas debido al agua infiltrada por las lluvias (Iverson, 1997; Nagarajan y Khire, 1998; AlcántaraAyala et al., 2001; Vilímek et al., 2006; Comegna et al., 2007; Okada et al., 2008) y, en algunos casos, por los sismos (Okada et al., 2008; Schneider, 2009). Previo a un movimiento de remoción de masa, este material se comporta como sólido pero, una vez que se inicia el movimiento de remoción, el material removido en muchos casos tiene un comportamiento líquido donde el flujo es no uniforme y no estacionario. En este caso, el tipo de evento de remoción de masa se denomina flujo hiperconcentrado de detritos. Existen algunas investigaciones en donde se analiza la dinámica de este tipo de movimientos de remoción de masa (Iverson, 1997; Chen y Lee, 2000; Fraccarollo y Papa, 2000; Denlinger e Iverson, 2001; Chen et al., 2005, Naef et al., 2006; Rosatti y Fraccarollo, 2006; Bateman et al., 2007; Mambretti et al., 2008; Wang et al., 2008). En particular, Iverson (1997), en su excelente revisión menciona que la mayoría de las investigaciones que estudian este tipo de fenómenos consideran condiciones de flujo uniforme y estacionario, lo cual lleva a conclusiones que lejanamente se aproximan a las situaciones observadas en este tipo de eventos. No obstante, existen muy pocos trabajos que se enfocan en analizar cuáles son los parámetros físicos que se modifican en una zona susceptible a la generación de flujo de detritos, en los momentos previos a la iniciación y que pueden fungir como indicadores de la ocurrencia. Chien-Yuan et al. (2005) realizaron un análisis histórico de la duración de las lluvias y lo relacionaron con la ocurrencia de los movimientos de remoción de masa. Estos autores encontraron que se presentan con mayor frecuencia una hora después del máximo de intensidad de las lluvias, por lo que implementaron un sistema de monitoreo de dicha intensidad por región para alertar a la población de la proximidad de un derrumbe en la isla de Taiwán. Sultanov y Khusanov (2001) mencionan que el estado de esfuerzos y deformación se ve afectado por el aumento de humedad en suelos propensos a asentamientos, lo que trae como consecuencia una mayor deformabilidad del suelo, provocado a partir de la disminución en la cohesión y la fricción interna del suelo. Estos autores proponen ecuaciones de estado en suelos propensos a asentamientos en función del contenido de humedad, incluyendo un modelo para la compresión volumétrica a presión constante, considerando un parámetro viscoso, la condición de plasticidad y relaciones que caracterizan la deformación bajo corte. Esto es, modelos de deformación elasto-plástica y elasto-visco-plástica.
A partir de estos parámetros, construyeron una función de plasticidad o cedencia que decrece exponencialmente respecto al contenido de humedad, tomando en cuenta la dependencia de la fuerza cohesiva y del coeficiente angular de fricción interna.

Uno de los indicadores más importantes de la proximidad de un derrumbe generado por lluvias es el esfuerzo de cedencia que se define como el esfuerzo mínimo para que un material empiece a fluir. Por lo tanto, para que un flujo de detritos tenga lugar, puede ocurrir que el esfuerzo de cedencia sea rebasado por el aumento de carga del agua infiltrada y/o que este esfuerzo disminuya debido a la presencia del agua que se infiltra generando una variación en la presión de poro (Juárez-Badillo y Rico-Rodríguez, 2002). Para determinar el esfuerzo de cedencia en función del contenido de humedad a partir del modelo de Sultanov y Khusanov (2001), se requiere la determinación de las curvas de esfuerzo-deformación, el coeficiente angular de fricción interna y la fuerza cohesiva para cada zona susceptible, lo que implica dificultades para determinarlo in situ, pues estos parámetros se obtienen a partir de pruebas de laboratorio. No obstante, el esfuerzo de cedencia puede calcularse a partir de la relación propuesta por Pashias et al. (1996), que considera la realización de una prueba de asentamiento en geometría cilíndrica de bajo costo. Estos autores mostraron que la prueba de asentamiento genera excelentes resultados al determinar el esfuerzo de cedencia en suspensiones minerales de barro rojo, circón y titanio. Esta técnica también ha sido utilizada para caracterizar arcillas (Caldiño- Villagómez et al., 2006; Sánchez-Cruz et al., 2006; Schatzmann et al., 2009), por lo que es posible realizar un estudio de la variación del esfuerzo de cedencia en función del contenido de agua para suelos arcillosos. Esta correlación entre el esfuerzo de cedencia y el contenido de agua permite evaluar la susceptibilidad de eventos de remoción de masa en una zona a partir del aumento de agua que se infiltra en el suelo por las lluvias, así como también permite determinar el valor del esfuerzo de cedencia para el cual ha ocurrido un flujo de detritos hiperconcentrado.

El presente trabajo analiza el esfuerzo de cedencia en función del contenido de agua a partir de una prueba de asentamiento realizada en laboratorio. Particularmente, se analiza la variación del esfuerzo de cedencia en función del contenido de agua para una muestra de suelo arcilloso que pertenece a una zona susceptible a flujo de detritos hiperconcentrado en el Valle de México.

El suelo arcilloso estudiado en este caso es de un lote que pertenece al cerro de Xico que se localiza en las coordenadas geográficas latitud norte $19^{\circ} 15^{\prime} 27^{\prime \prime}$ y longitud oeste $98^{\circ} 56^{\prime} 33^{\prime \prime}$ y pertenece al municipio de Valle de Chalco Solidaridad. Esta región es principalmente plana, con excepción del cerro del Xico y el Marqués, que son volcanes monogenéticos, y se localiza en la zona de lo que fue un antiguo lago en tiempos pleistocénicos, el cual se azolvó por deposición lacustre, eólica y aluvial (INAFEDSEGOB, 2010). Los tipos de suelos que abundan en esta 
zona son el franco limoso, franco arcilloso y franco arenoso con una resistencia a la compresión promedio de $2 \mathrm{~T} / \mathrm{m}^{2}$ (CAEM, 2009). La precipitación media anual es de $600 \mathrm{a}$ $700 \mathrm{~mm}$ de agua.

En esta zona, el 6 de septiembre de 2009, se presentó una precipitación pluvial extraordinaria de $27.4 \mathrm{~mm}$ (SMN, 2009) que originó la bajada de aguas broncas del cerro de Xico y acarreo de tierra (CAEM, 2009). Se eligió el suelo arcilloso de esta región para el presente estudio debido a que se considera una zona vulnerable a deslaves (Figura 1) y en específico de su potencialidad de generar flujo de detritos hiperconcentrados según el evento documentado.

\section{Experimentación}

\subsection{Prueba de asentamiento}

La prueba de asentamiento consiste en llenar un cilindro de altura y radio conocidos con el material al cual se le quiere determinar el esfuerzo de cedencia. Posteriormente, se levanta el cilindro permitiendo que el material se colapse bajo su propio peso (como se muestra en la Figura 2). La diferencia entre la altura inicial y la altura final se denomina altura de asentamiento(s).

La expresión propuesta por Pashias et al. (1996), está dada por la siguiente expresión:

$$
s^{\prime}=1-2 \tau_{y}^{\prime}\left[1-\ln \left(2 \tau_{y}^{\prime}\right)\right]
$$

Ésta relaciona dos cantidades, la altura de asentamiento normalizada $s^{\prime}=\frac{s}{H}$ y el esfuerzo de cedencia normalizado $\tau_{y}^{\prime}=\frac{\tau_{y}}{\rho g H}$ , donde $\mathrm{H}$ es la altura del cilindro, $\tau_{y}$ es el esfuerzo de cedencia, $\rho$ es la densidad de la muestra y $g$ $=9.81 \mathrm{~m} / \mathrm{s}^{2}$. Entonces, basta con determinar la altura de asentamiento normalizada, sustituirla en la ecuación (1) y resolver esta ecuación para $\tau^{\prime}$. Una expresión simplificada para la ecuación (1) puede obtenerse empleando la serie:

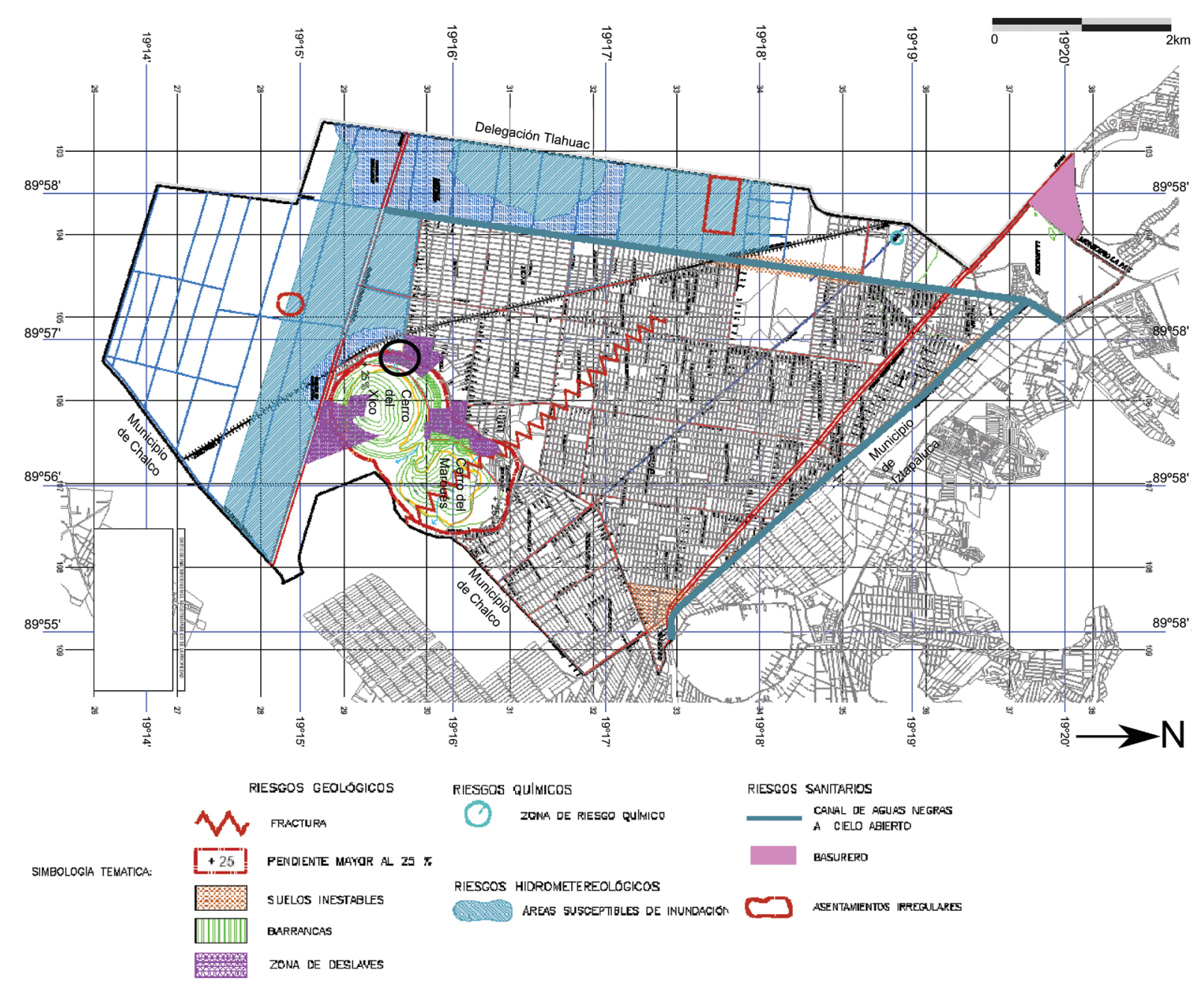

Figura 1. Mapa de riesgos del municipio de Valle de Chalco, proporcionado por la Dirección de Protección Civil y Bomberos local (2008). El óvalo negro muestra la zona de estudio del cerro de Xico en donde se obtuvo la muestra. 


$$
\ln 2 \tau_{y}^{\prime}=\left(2 \tau_{y}^{\prime}-1\right)+\frac{1}{2}\left(2 \tau_{y}^{\prime}-1\right)^{2}+\frac{1}{3}\left(2 \tau_{y}^{\prime}-1\right)^{3}+\ldots
$$

Ésta es válida para valores de $\tau_{y}^{\prime}$ que pertenecen al intervalo $\frac{1}{2} \geq \tau_{y}^{\prime}>0$ por lo que, a partir de una aproximación a primer orden, se tiene una expresión del esfuerzo de cedencia $\tau_{y}^{\prime}$ :

$$
\tau^{\prime}{ }_{y}=\frac{1}{2}-\frac{1}{2} \sqrt{s^{\prime}}
$$

Es de hacer notar que cuando s' $=0$, no existe asentamiento y el esfuerzo de cedencia normalizado es igual a $1 / 2$, mientras que cuando $\mathrm{s}^{\prime}=1$, conduce a la ausencia de cedencia y el material fluye, es decir, el esfuerzo de cedencia es igual a cero.

Finalmente, el esfuerzo de cedencia está determinado a partir de la ecuación (3) multiplicada por la cantidad $\rho g H$, como:

$$
\tau_{y}=\rho g H\left[\frac{1}{2}-\frac{1}{2} \sqrt{\frac{s}{H}}\right]
$$

\subsection{Características de la muestra}

Las muestras utilizadas en este trabajo fueron tomadas superficialmente del cerro de Xico a una profundidad $\leq 0.50$ $\mathrm{m}$. La Figura 3a muestra una imagen del lugar de donde fueron recolectadas, y la Figura $3 \mathrm{~b}$ muestra una imagen de la arcilla in situ.

Las muestras colectadas se tamizaron con una malla del número 8 (lado $3.1 \mathrm{~mm}$ ) para eliminar basura y rocas de mayor tamaño. La densidad relativa en seco fue de 1.779. El límite húmedo fue del $33.6 \%$, el límite plástico fue $44.1 \%$, mientras que límite líquido fue del 44.8 $\%$, los contenidos gravimétricos fueron de $\mathrm{u}=0.336$, 0.0441 y 0.448 , respectivamente. La Figura 4 muestra la distribución de tamaños de partícula, donde más del $50 \%$ de los tamaños de partículas son finos y menores a 0.045 $\mathrm{mm}$, por lo que existe mayor plasticidad y, por lo tanto, un mayor potencial de desplazamiento del material, un $15 \%$ cae en el intervalo de 0.050 a $0.090 \mathrm{~mm}$, el $33 \%$ está en el intervalo comprendido de 0.200 a $1.000 \mathrm{~mm}$, y el restante entre 1.000 y $2.000 \mathrm{~mm}$.

Se realizaron análisis de difracción de rayos $\mathrm{X}$ para determinar los minerales del suelo arcilloso, encontrándose cristobalita $\left[\mathrm{SiO}_{2}\right]$, tridimita $\left[\mathrm{SiO}_{2}\right]$, montmorillonita $\left[\mathrm{Al}_{2} \mathrm{O}_{5} \bullet 4 \mathrm{SiO}_{2} \bullet 4 \mathrm{H}_{2} \mathrm{O}\right]$, bentonita $\left[(\mathrm{Al}, \mathrm{Mg}) 8\left(\mathrm{Si}_{4} \mathrm{O}_{10}\right) 4(\mathrm{OH})_{8} \cdot 12 \mathrm{H}_{2} \mathrm{O}\right]$ y nontronita $\left[\mathrm{H}_{4} \mathrm{Fe}_{2} \mathrm{Si}_{2} \mathrm{O}_{9}\right]$ (Sánchez-Cruz, 2008).

Se prepararon muestras de $0.3 \mathrm{~kg}$ agregando la cantidad de agua necesaria para posteriormente mezclarlas. Esto se hizo para concentraciones porcentuales en peso de agua de $23,24,25,26,27,28,29$ y $30 \%$. Estas concentraciones se consideraron a prueba y error, ya que a concentraciones menores al $23 \%$ en peso el mezclado de las muestras no era

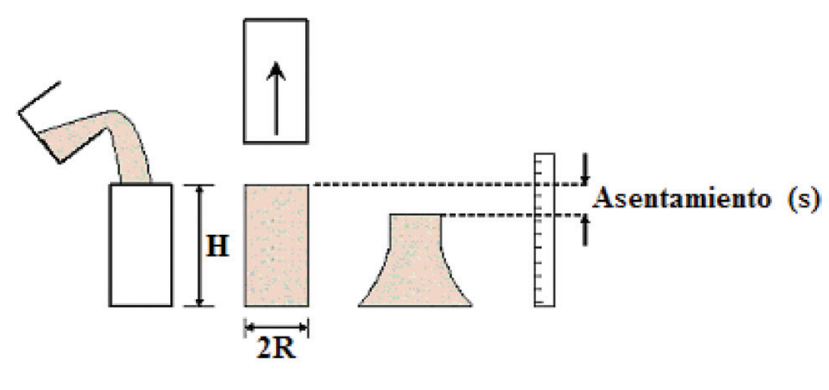

(a) (b) (c) (d)

Figura 2. Prueba de asentamiento, a) llenado del cilindro, b) se retira el cilindro, c) asentamiento de la muestra bajo su propio peso, d) determinación de la altura de asentamiento (s). H y R corresponden a la altura y radio del cilindro respectivamente.
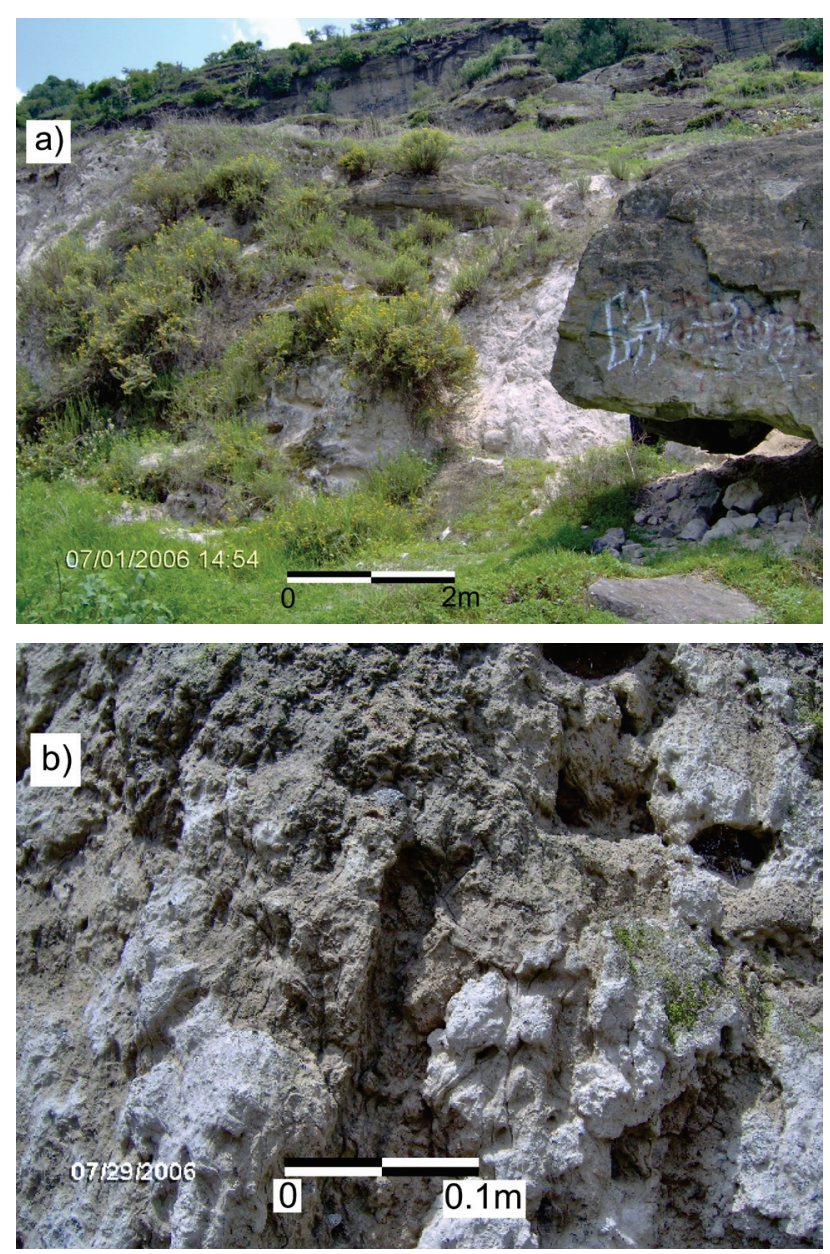

Figura 3. Localización de la muestra de arcilla extraída del cerro de Xico. a) Imagen de la zona, b) detalle de la muestra in situ.

homogéneo, resultado de su alta capacidad de retención de agua, por lo que sólo se generaron algunos grumos húmedos en ellas y el asentamiento fue prácticamente nulo. Es de esperarse entonces que a concentraciones menores no se dé un movimiento de remoción inducido por lluvias. Por otra parte, a contenidos de agua mayores al $30 \%$ en peso, 


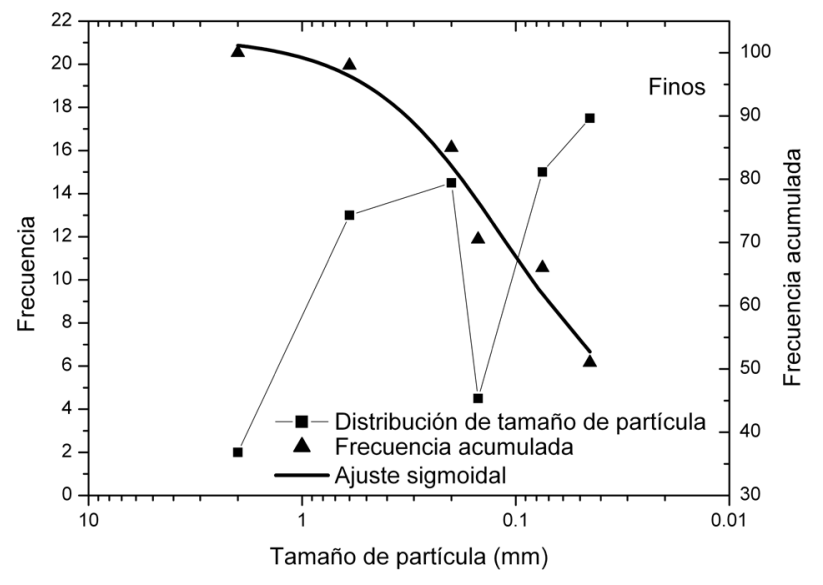

Figura 4. Distribución de tamaños de partícula de la muestra del cerro de Xico.

la muestra se comportó como un fluido con un esfuerzo de cedencia demasiado bajo, por lo que a estas concentraciones se tendría una situación de flujo de detritos para este suelo arcilloso.

Se determinó la densidad para cada concentración de la muestra estudiada a partir de la medida de la masa y el volumen. La masa se midió usando una báscula electrónica marca Sartorius modelo CP 3202P, mientras que el volumen se determinó utilizando una probeta graduada. En la Tabla 1 se muestra la densidad relativa para cada concentración utilizada.

\subsection{Medición del esfuerzo de cedencia}

Las pruebas de asentamiento se realizaron empleando un dispositivo construido tal como se muestra en la Figura 5. El dispositivo consiste de una base y un cilindro. La base tiene una plataforma de vidrio nivelada y un vernier fijo, al vernier se le ajustó una extensión en el nonio para medir la altura del asentamiento. El cilindro que se utilizó es de PVC de altura $\mathrm{H}=0.047 \mathrm{~m}$, diámetro $\mathrm{D}=0.037 \mathrm{~m}$ y con una razón $\mathrm{H} / \mathrm{D}=1.28$, que es una de las recomendadas por Pashias et al. (1996) para estas pruebas.

Para las mediciones del asentamiento, se vertió la arcilla en el cilindro hasta llenarlo, y se retiró manualmente permitiendo que la muestra se asentara bajo su propio peso. Posteriormente, se midió la altura final del asentamiento $s$ con ayuda del vernier después de 40 segundos (también, se midieron las alturas a un tiempo de 180 segundos y los resultados fueron similares). Este tiempo se eligió debido a que a tiempos posteriores (mayores a 180 segundos) se presentaron problemas de evaporación, mientras que para tiempos menores a 20 segundos la muestra continuaba asentándose. Mediante la Ecuación 3 se calculó el esfuerzo de cedencia normalizado. La Figura 6 ejemplifica un asentamiento.
Tabla 1. Distribución de densidades relativas de las diferentes muestras preparadas.

\begin{tabular}{ccccccccc}
\hline $\begin{array}{c}\text { Concentración } \\
\text { porcentual }\end{array}$ & 23 & 24 & 25 & 26 & 27 & 28 & 29 & 30 \\
$\begin{array}{c}\text { Densidad } \\
\text { relativa } \\
( \pm 0.014)\end{array}$ & 1.477 & 1.457 & 1.448 & 1.439 & 1.431 & 1.417 & 1.4088 & 1.402 \\
\hline
\end{tabular}

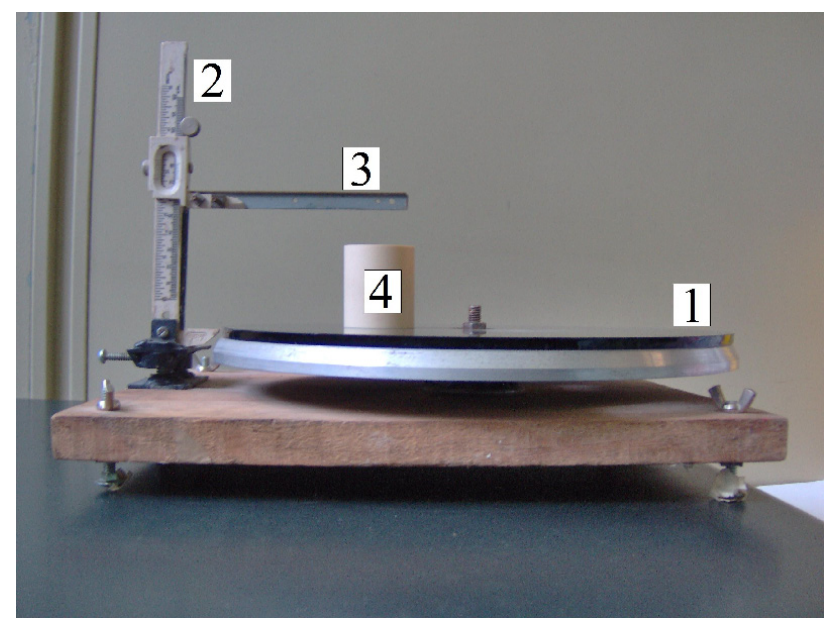

Figura 5. Dispositivo construido para las pruebas de asentamiento. 1) Plataforma de vidrio, 2) vernier, 3) extensión en el nonio y 4) cilindro utilizado.

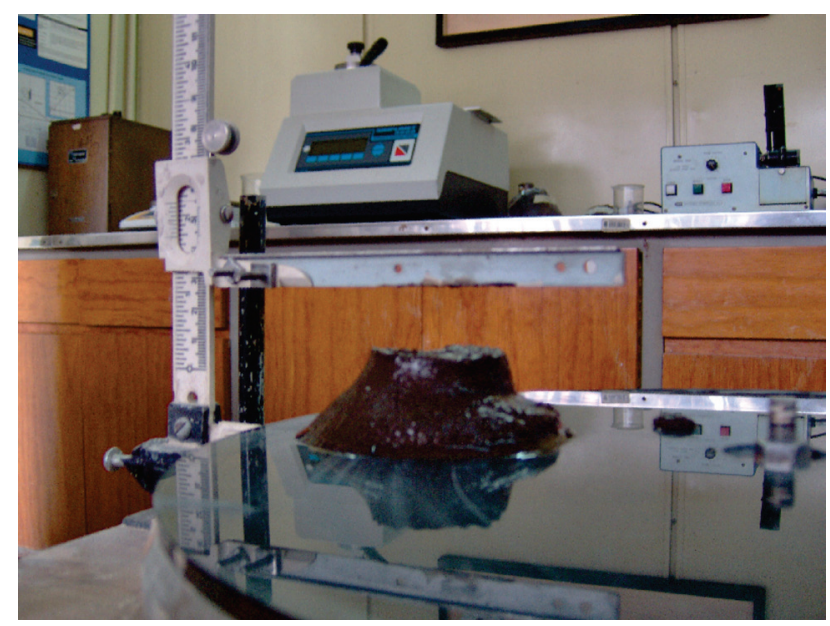

Figura 6. Determinación del asentamiento empleando el dispositivo construido.

\section{Resultados y Discusión}

En la Figura 7 se muestra la gráfica del asentamiento normalizado (eje vertical izquierdo) y el esfuerzo de cedencia normalizado (eje vertical derecho) en función del contenido en peso de agua para la muestra bajo estudio. Se aprecia que para concentraciones de agua del 23,24 y 25 
$\%$ el valor del asentamiento se incrementa gradualmente al aumentar el contenido de agua. Estos valores son menores a 0.3 que corresponde aproximadamente al $30 \%$ de la altura total. Sin embargo, a $26 \%$ de agua existe un cambio abrupto en el asentamiento, alcanzando un valor mayor a 0.75 , es decir, mayor al $75 \%$ de la altura total. Esto indica que el incremento de agua ha realizado un cambio importante en las propiedades de la arcilla. Particularmente, esta variación indica que la muestra ha dejado de ser una suspensión sólida y pasa a ser una suspensión líquida, por lo que bajo estas condiciones la arcilla fluye provocando, en condiciones in situ, un movimiento de remoción de tierra.

Los resultados obtenidos son consistentes con el comportamiento esperado para este suelo arcilloso debido a la presencia de montmorillonita y bentonita, ya que son minerales de altas propiedades plásticas y capaces de absorber cantidades de agua relativamente grandes entre sus capas adyacentes, cambiando su distancia basal desde $10 \AA$ hasta $20 \AA$ (Singer y Singer, 1979). Una vez que se alcanza su estado de saturación crítico, es de esperarse un cambio drástico en algunas de las propiedades físicas, como lo representa el aumento abrupto del asentamiento. Este comportamiento también se observa en el esfuerzo de cedencia normalizado en función de la concentración porcentual de agua. En este caso los esfuerzos normalizados a bajas concentraciones en el intervalo 23-25\% decrecen gradualmente conforme se incrementa la concentración de agua pero siempre son superiores al 0.2. Una vez que se llega a concentraciones de agua mayores o iguales al 26 $\%$, el esfuerzo normalizado disminuye por abajo del 0.06 , es decir menores al $6 \%$. Para las concentraciones de agua entre el $26 \%$ y $30 \%$, el esfuerzo de cedencia disminuyó dramáticamente, por lo que la muestra cede fácilmente debido a que el esfuerzo en la columna de asentamiento es

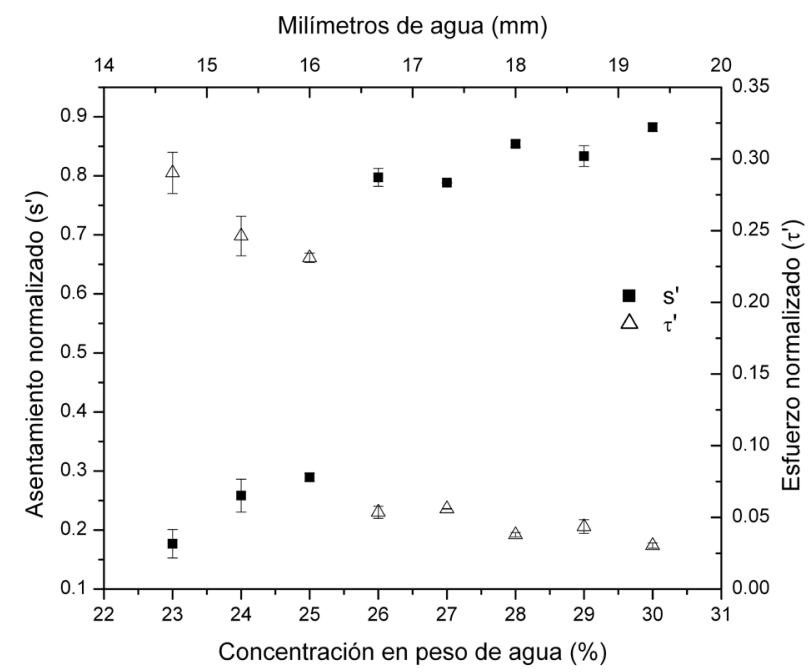

Figura 7. Gráfica del asentamiento y el esfuerzo de cedencia (eje vertical derecho) normalizados en función de la concentración porcentual en peso de agua. Nótese el cambio abrupto para concentraciones en el intervalo $25-26 \%$ en peso de agua. mucho mayor que el esfuerzo de cedencia. Ello evidencia que se generaría un flujo o movimiento de remoción de tierra, como se había mencionado anteriormente.

En la Figura 8 se muestra el esfuerzo de cedencia calculado a partir de la Ecuación 4, en función de la concentración porcentual de agua con sus correspondientes barras de error. Se puede observar que para concentraciones de agua en el intervalo 23-25\%, el esfuerzo de cedencia disminuye gradualmente en el intervalo de $200 \mathrm{~Pa}$ a 150 $\mathrm{Pa}$, como es de esperarse debido a la poca concentración de agua. Por otro lado, en el intervalo crítico de $25-26 \%$ se presenta el cambio más abrupto en el esfuerzo de cedencia que va de un valor de $153 \mathrm{~Pa}$ a $35.5 \mathrm{~Pa}$. Si recordamos que la presión de poro representa a la presión de agua que llena los espacios vacíos, el suelo se vuelve inestable debido a que las partículas pierden cohesión entre sí, lo que resulta en la disminución dramática en el esfuerzo de cedencia. Para concentraciones de agua mayores que el $26 \%$, la tendencia general es a disminuir entre los valores de $38.3 \mathrm{~Pa}$ y $19 \mathrm{~Pa}$.

El comportamiento observado en la Figura 8, puede compararse con la función de plasticidad propuesta por Sultanov y Khusanov (2001), donde ésta presenta un comportamiento exponencial decreciente al incremento de humedad. No obstante, dicha función es incapaz de mostrar el cambio abrupto observado en este suelo arcilloso. Un comportamiento de la cedencia cercanamente exponencial en función del contenido de humedad ha sido observado recientemente por Nieto-Zepeda et al. (2008) para suelos arcillosos, aunque también se presenta un salto abrupto. Es probable que el comportamiento exponencial visto esté relacionado con la composición química de la arcilla.

En el eje horizontal superior de las Figuras 7 y 8, se incluye una relación de la concentración de agua y la cantidad de milímetros de agua de lluvia. Esta se calculó

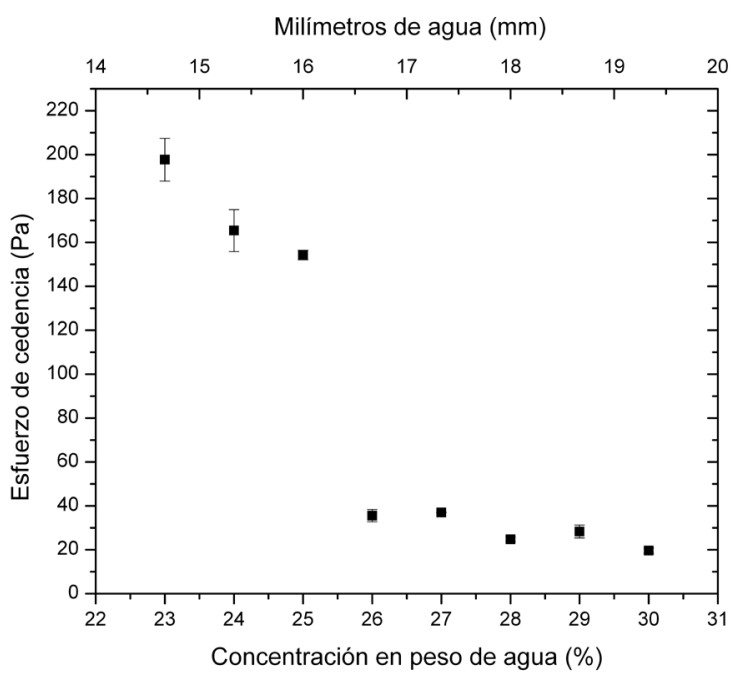

Figura 8. Esfuerzo de cedencia en función de la concentración en peso de agua. El eje horizontal superior relaciona los milímetros de agua con la concentración en peso de agua. 
a partir de dividir el volumen de agua que corresponde al volumen de muestra (para una concentración fija) contenida en el cilindro entre el área del cilindro, suponiendo que dicha cantidad de agua fue colectada en el cilindro debido a las precipitaciones pluviales. Aunque esta suposición es burda, se tiene una idea aproximada de los milímetros mínimos necesarios para la ocurrencia de un flujo de detritos hiperconcentrado. Hasta donde se sabe, no se tienen datos en la literatura de la absorción de agua pluvial en la zona susceptible a este tipo de eventos que permitan mejorar esta aproximación. Para hacerlo, se debe tomar en cuenta que sólo parte del agua pluvial es absorbida por el suelo, mientras que la restante se transporta por vertientes hacia ríos o coladeras. En este caso, la región crítica en donde el esfuerzo decrece abruptamente se localiza entre 16 y $17 \mathrm{~mm}$ de agua. Es de hacer notar que estos valores están por debajo de la precipitación pluvial extraordinaria $(27.4 \mathrm{~mm})$ ocurrida el 6 de septiembre de 2009 en esta zona. No obstante, en el evento mencionado no se tiene la certeza de que el nivel de humedad crítico en la tierra se hubiera alcanzado.

\section{Conclusiones}

En el presente trabajo se determinó el esfuerzo de cedencia a partir de una prueba de asentamiento en función del contenido en peso de agua para un suelo arcilloso susceptible a generar un flujo de detritos hiperconcentrado a causa de las precipitaciones pluviales en el Cerro de Xico. Se obtuvo el gráfico asentamiento-concentración de agua y el gráfico esfuerzo de cedencia-concentración, y se estableció una relación directa entre el esfuerzo de cedencia y los milímetros de agua a partir de la concentración en peso de agua, para el suelo arcilloso. Se encontró un intervalo crítico donde el esfuerzo disminuye abruptamente para una concentración en peso de agua en el intervalo 25-26 \% (16$17 \mathrm{~mm}$ de agua aproximadamente), que es el punto donde se considera se tienen condiciones críticas para que ocurra un flujo de detritos hiperconcentrado. Además, se sugiere que dicha prueba puede ser empleada in situ, teniendo así un estudio de la variación del esfuerzo de cedencia en función del contenido de agua en cada región de interés.

\section{Agradecimientos}

Los autores agradecen a Aline Concha Dimas, Gilberto Salgado Maldonado, Ignacio Caldiño Villagómez e Isaac Bonola Alonso, por sus atinados comentarios vertidos durante la revisión del presente trabajo.

\section{Referencias}

Alcántara-Ayala, I., Echavarría-Luna, A., Gutiérrez-Martínez, C., Domínguez-Morales, L., Noriega-Rioja, I., 2001, Inestabilidad de laderas: México, D.F., Cenapred-Secretaría de Gobernación, 36 p. Bateman, A., Medina, V., Hürlimann, M., Velasco, D., 2007, Modelo bidimensional para simulación de flujos detríticos: FLATModel. Aplicación a una cuenca del Pirineo Catalán: Ingeniería Hidráulica en México, 22, 5-20.

Bisson, M., Favalli, M., Fornaciai, A., Mazzarini, F., Isola, I., Zanchetta, G., Pareschi, M. T., 2005, A rapid method to assess fire-related debris flow hazard in the Mediterranean region: An example from Sicily (southern Italy): International Journal of Applied Earth Observation and Geoinformation, 7, 217-231.

Caldiño-Villagómez, I.A., Bonola-Alonso, I., Salgado-Maldonado, G., 2006, Estudio experimental del esfuerzo de cedencia con relación al flujo de lodos y debris (resumen), en Memorias del XXII Congreso Latinoamericano de Hidráulica: Guayana, Venezuela, Asociación Internacional de Ingeniería e Investigaciones Hidro-Ambientales, 8.

Collins, T.K., 2008, Debris flows caused by faillure of fill slopes: early detection, warning, and loss prevention: Landslides, 5, 107-120.

Comisión de Agua del Estado de México (CAEM), 2009, Atlas de inundaciones del municipio de Valle de Chalco solidaridad, 2009, (en línea): Comisión de Agua del Estado de México - Gobierno del Estado de México, disponible en <http://qacontent.edomex.gob.mx/ idc/groups/public/documents/edomex_archivo/caem_pdf_chalcoso. pdf $>$ Consultado 8 de noviembre de 2010.

Comegna, L., Picarelli, L., Urciuoli G., 2007, The mechanics of mudslides as a cyclic undrained-drained process: Landslides, 4, 217-232.

Chen, H., Lee, C.F., 2000, Numerical simulation of debris flows: Canadian Geotechnical Journal, 37, 146-160.

Chen, J., He, Y.P., Wei, F.Q., 2005, Debris flow erosion and deposition in Jiangjia Gully, Yunnan, China: Environmental Geology, 48, 771-777.

Chien-Yuan, C., Tien-Chen, C., Fan-Chieh, Y., Wen-Hui, Y., Chun-Chieh, T., 2005, Rainfall duration and debris-flow initiated studies for realtime monitoring: Environmental Geology, 47, 715-724.

Denlinger, R.P., Iverson, R.M, 2001, Flow of variably fluidized granular masses across three-dimensional terrain 2 . Numerical prediction and experimental test: Journal of Geophyscal Research, 106, 553-566.

Dirección de Protección Civil y Bomberos del Municipio de Valle de Chalco Solidaridad, 2008, Atlas de riesgo de Valle de Chalco: Toluca, Estado de México, Folleto Técnico, 106 p.

Fraccarollo, L., Papa, M., 2000, Numerical simulation of real debris-flow events: Physics and Chemistry of the Earth, Part B: Hydrology, Oceans and Atmosphere, 25, 757-763.

Instituto para el federalismo y el desarrollo municipal, Secretaria de Gobernación (INAFED-SEGOB), 2010, Enciclopedia de los municipios y delegaciones de México, Estado de México, Valle de Chalco Solidaridad (en línea): México, D. F., INAFED-SEGOB, disponible en <http://e-local.gob.mx/work/templates/enciclo/ EMM15mexico/> Consultado 10 de enero de 2011

Iverson, R.M., 1997, The physics of debris flows: Reviews of Geophysics, 35, 245-296.

Juárez-Badillo, E., Rico-Rodríguez, A., 2002, Mecánica de suelos Tomo 1, Fundamentos de la mecánica de suelos: México, D. F., Limusa, 642 p.

Mambretti, S., Larcan, E., De Wrachien, D., 2008, 1D modelling of dam-break surges with floating debris: Biosystems Engeneering, 100, 297-308

Naef, D., Rickenmann, D., Rutschmann, P., McArdell, B.W., 2006, Comparison of flow resistance relations for debris flows using a one-dimensional finite element simulation model: Natural Hazards and Earth Systems Sciences, 6, 155-165.

Nagarajan, R., Khire M.V., 1998, Debris slides of Varandh Ghat, west coast of India: Bulletin of Engineering Geology and the Environment, $57,59-63$.

Nieto-Zepeda, K.E., Méndez-Sánchez, A.F., Pérez-Trejo, L., PaniaguaMercado, A.M, 2008, Análisis del derrumbe ocurrido en Teziutlán, Puebla, a partir de la determinación del esfuerzo de cedencia (resumen), en LI Congreso Nacional de Física: Zacatecas, Zacatecas, México, Sociedad Mexicana de Física, 114.

Okada, Y., Ochiai, H., Kurokawa, U., Ogawa, Y., Asano, S., 2008, A channelised long run-out debris slide triggered by the Noto Hanto earthquake in 2007, Japan: Landslides, 5, 235-239. 
Pashias, N., Boger, D.V., Summers, J., Glenister, D.J., 1996, A fifty cent rheometer for yield stress measurements: Journal of Rheology, 40, 1179-1190.

Rosatti, G., Fraccarollo, L., 2006, A well-balanced approach for flows over mobile-bed with high sediment-trasport: Journal of Computational Physics, 220, 312-338.

Sánchez-Cruz, P., 2008, Análisis del esfuerzo de cedencia de suelos arcillosos como posible indicador de un derrumbe: México, D. F., Instituto Politécnico Nacional, Tesis de licenciatura, $51 \mathrm{p}$.

Sánchez-Cruz, P., Rivera-Martínez, I., Pérez-Trejo, L., Méndez-Sánchez, A.F., 2006, Determinación del esfuerzo de cedencia a partir de una prueba de asentamiento (resumen), en XLIX Congreso Nacional de Física: San Luis Potosí, S.L.P., México, Sociedad Mexicana de Física, 88.

Schatzmann, M., Bezzola, G.R., Minor, H.-E., Windhab, E.J., Fischer, P., 2009, Rheometry for large-particulated fluids: analysis of the ball measuring system and comparison to debris flow rheometry: Rheologica Acta, 48, 715-733.

Schneider, J.F., 2009, Seismically reactivated hattian slide in Kashmir, Northern Pakistan: Journal of Seismology, 13, 387-398.

Servicio Meteorológico Nacional (SMN), 2009, Análisis mensual de precipitación por entidad federativa (en línea): México, D.F., SMNConagua-Semarnat, disponible en $<\mathrm{http}$ ///smn.conagua.gob.mx/ index.php?option $=$ com_content $\&$ view $=$ article $\& i d=12 \&$ Itemid $=77>$ Consultado 27 de enero de 2011 .
Singer, F., Singer, S.S., 1979, Cerámica industrial Vol. 1: Bilbao, España, URMO, $762 \mathrm{p}$.

Sultanov K.S., Khusanov B.É., 2001, State equations for soils prone to slump-type settlement with allowance for degree of wetting: Soil Mechanics and Foundation Engineering, 38, 80-86.

VanDine, D.F., Rodman, R.F., Jordan, P., Dupas, J., 2005, Kuskonook Creek, an example of a debris flow analysis: Landslides, 2, 257-265.

Vilímek, V., Klimés, J., Vicko, J., Carreño R., 2006, Catastrophic debris flows near Machu Picchu village (Aguas Calientes), Peru: Environmental Geology, 50, 1041-1052.

Wang, C., Li, S., Esaki, T., 2008, GIS-based two-dimensional numerical simulation of rainfall-induced debris flow: Natural Hazards and Earth Systems Sciences, 8, 47-58.

Manuscrito recibido: Diciembre 15, 2009

Manuscrito corregido recibido: Octubre 10, 2010.

Manuscrito aceptado: Noviembre 5, 2010. 